\title{
Discovery of secondary metabolites in an extractive liquid-surface immobilization system and its application to high-throughput interfacial screening of antibiotic-producing fungi
}

\author{
Shinobu Oda, Arisa Kameda, Masanori Okanan, Yusuke Sakakibara and Shinichi Ohashi
}

An extractive liquid-surface immobilization (Ext-LSI) system, which consists of a hydrophobic organic solvent (an upper phase), a fungal cell-ballooned microsphere layer (a middle phase) and a liquid medium (a lower phase), is a unique interfacial cultivation system for fungi. The fungal cells growing at the interface between the organic and aqueous phases efficiently produce hydrophobic metabolites, which are continuously extracted into the organic phase, and/or hydrophilic metabolites that migrate into the aqueous phase without carbon catabolite repression and product and/or feed-back inhibitions. Application of the system to fermentation of Penicillium multicolor IAM 7153 and Trichoderma atroviride AG2755-5NM398 afforded remarkably different profiles of secondary metabolites in the organic phase compared with those in an aqueous phase in traditional submerged cultivation (SmC). Various hydrophobic metabolites exhibiting unique UV-visible spectra were accumulated into the organic phase. The system was applied to a novel interfacial screening system of antibiotic-producing fungi. Compared with the SmC, the interfacial cultivation system exhibited some interesting and important advantages, such as the higher accumulation of hydrophobic secondary metabolites, the lack of requirement for shaking and troublesome solvent extraction, and the small scale of the vessels (medium, $5 \mathrm{ml}$; dimethylsilicone oil, $1 \mathrm{ml}$ ), as well as the significantly different metabolite profiles. The interfacial screening system yielded a high incidence of antimicrobial activity, with $21.9 \%$ of the fungi tested exhibiting antifungal activity against Pichia anomala NBRC 10213. This novel interfacial high-throughput screening approach has the potential to discover new biologically active secondary metabolites even from strains previously found to be unproductive.

The Journal of Antibiotics (2015) 68, 691-697; doi:10.1038/ja.2015.59; published online 13 May 2015

\section{INTRODUCTION}

The threat of multidrug-resistant pathogens such as Mycobacterium tuberculosis, Staphylococcus aureus and Pseudomonas aeruginosa is on the rise with frequent recent reports of poorly controlled hospital infections and community-associated epidemics. ${ }^{1,2}$ At the same time, research and development into new antibiotics by the pharmaceutical industry has decreased very significantly. New initiatives to discover and develop antibiotics effective against multidrug-resistant bacteria and fungi, and viruses are urgently needed.

Many secondary metabolites produced by microorganisms, mainly actinomycetes and fungi, have been isolated, identified and evaluated as antimicrobial agents over the last seven decades. Although actinomycetes, especially Streptomyces spp., have afforded many important antibiotics, such as tetracyclines, cephalosporins, aminoglycosides and macrolides, the potential of fungi as antibiotic producers has not been fully realized given their incomparably greater diversities of genus, species and gene compared with actinomycetes. ${ }^{3,4}$ Especially, fungi isolated from unusual environments such as endophytic, ${ }^{5,6}$ entomophagous ${ }^{7,8}$ and marine-derived fungi ${ }^{9,10}$ have lately attracted considerable attention as producers of biologically active secondary metabolites.

As for the submerged cultivation (SmC) of fungi, it is well known that the pelletted form is a favorable morphology for most fungal fermentations because it enables higher mass and oxygen transfer and lower energy consumption for aeration and agitation compared with filamentous and clump forms. ${ }^{11,12}$ However, the control of fungal morphology to the pelletted form is generally troublesome because many factors such as medium composition and $\mathrm{pH}$, agitation and aeration rates, sparger and agitator structures, volume and style of inoculum must be optimized. ${ }^{13,14}$ It was also reported that some additives, such as polymers, surfactants, oils and $\mathrm{CaCO}_{3}$ particles, significantly affected fungal morphology and fermentation performance. ${ }^{15,16}$ Moreover, the pelletted form is not natural because almost all fungi exist as thick fungal mats on the surface of solids such as walls, rocks and stems of higher plants. The authors therefore expect that the fungal mat, as a natural fungal formation, will be a favored physiological state for the production of secondary metabolites. 


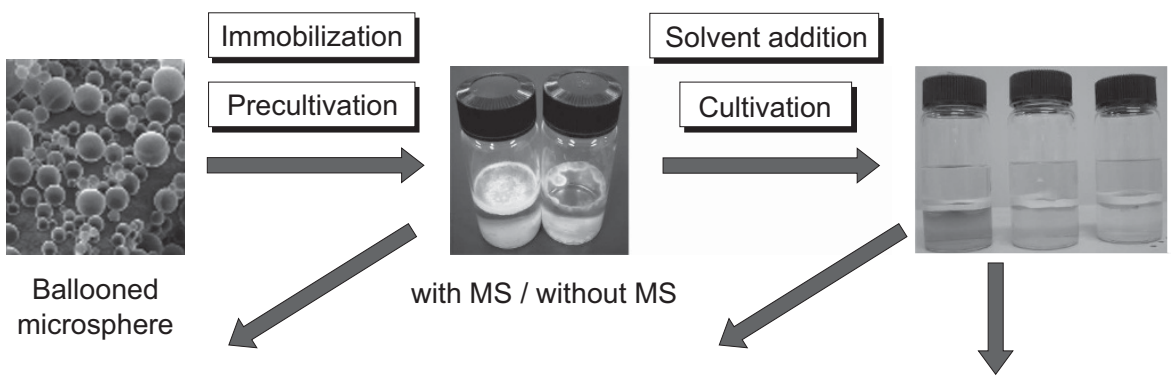

a Liquid-surface Immobilization (LSI) System

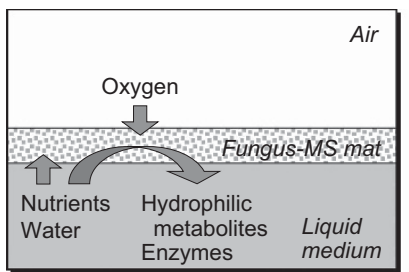

MS, ballooned microsphere b Liquid-liquid Interface Bioreactor (L-L IBR)
C Extractive Liquid-surface Immobilization (Ext-LSI) System

\begin{tabular}{|ll|}
\hline \multicolumn{2}{|c|}{ Oxygen } \\
\hline \\
$\begin{array}{l}\text { Hydrophobic } \\
\text { substrate }\end{array}$ & $\begin{array}{c}\text { Hydrophobic } \\
\text { product }\end{array}$ \\
\hline & Fungus-MS mat \\
\hline $\begin{array}{l}\text { Nutrients } \\
\text { Water }\end{array}$ & Liquid medium \\
\hline
\end{tabular}

Figure 1 A genealogy of three types of fungal cultivation systems using ballooned microsphere (MS). In all systems, fungal cells are trapped on a ballooned polyacrylonitrile MS layer formed on the surface of a liquid medium. The fungal cells grow and differentiate to vegetative and aerial hyphae, sporangia and spores during appropriate precultivation period to form a physically strong fungus-MS mat. In the LSI system (a), fungal cells produce hydrophilic metabolites and/or enzymes. The L-L IBR system (b) affords hydrophobic products via interfacial microbial transformation of hydrophobic substrates dissolved in a hydrophobic organic phase. In the Ext-LSI system (c), hydrophobic metabolites are produced via interfacial fermentation to accumulate into an organic phase. A full color version of this figure is available at The Journal of Antibiotics journal online.

Recently, we have developed three types of unique cultivation and application systems with a fungal mat, the liquid-surface immobilization (LSI) system, the liquid-liquid interface bioreactor, and the extractive liquid-surface immobilization (Ext-LSI) system (Figure 1a-c). In all systems, fungal cells suspended in a liquid medium are effectively floated onto the surface of a liquid medium by the aid of polyacrylonitrile ballooned microspheres and form a physically strong fungal mat together with numerous microspheres. Among the three systems, the LSI system (Figure 1a) has been applied to the production of some enzymes such as lipase, ${ }^{17}$ xylanase ${ }^{18}$ and $\beta$-glucosidase, ${ }^{19}$ and hydrophilic natural products such as pigments. In all cases, the LSI system afforded excellent productivity compared with SmC.

The liquid-liquid interface bioreactor (Figure 1b), an organicaqueous interfacial bioconversion system with fungal cells, has been applied to some microbial transformations, such as hydrolysis of an acetate ester, ${ }^{17,20}$ asymmetric reduction of an aromatic diketone, ${ }^{21}$ regio- and stereoselective hydroxylation of $n$-alkanes ${ }^{22-24}$ and regioand stereoselective epoxidation of a sesquiterpene. ${ }^{25}$ In all cases, lipophilic substrate dissolved in a hydrophobic organic solvent is efficiently converted to lipophilic product by an interfacial fungal mat. The excellent accumulation of conversion products was achieved compared with SmC. For example, the accumulation of (-)- $\beta-$ caryophyllene oxide of which the MIC against many fungi was $50-200 \mathrm{mgl}^{-1}$ in the aqueous phase reached $30 \mathrm{gl}^{-1}$ in the organic phase. $^{25}$

The Ext-LSI system (Figure 1c), an organic-aqueous interfacial fermentation system with fungal cells, has been applied to the production of a fungicidal secondary metabolite, 6-pentyl- $\alpha$-pyrone (6PP), by Trichoderma atroviride. ${ }^{26}$ In the system, product and/or feedback inhibition can be effectively alleviated because lipophilic metabolite produced by an interfacial fungal mat is spontaneously extracted from cells into a hydrophobic organic solvent. Fungicidal 6PP of which MIC ranged from 0.1 to $0.5 \mathrm{gl}^{-1}$ in aqueous phase against Trichoderma harzianum, Botrytis cinerea and Sclerotinia sp..$^{27,28}$ However, the Ext-LSI system with T. atroviride AG2755-5NM398 could accumulate $7.1 \mathrm{gl}^{-1}$ of $6 \mathrm{PP}$ in the organic phase. ${ }^{26}$

Recently, two unique phenomena of the Ext-LSI system were discovered. The addition of anion-exchange resin microparticles into an MS layer significantly enhanced the production of $6 \mathrm{PP}$ in a modified Ext-LSI system, ${ }^{29}$ and carbon catabolite repressions (CCRs) by glucose and fructose were effectively derepressed for the production of 6PP in the Ext-LSI system. It is expected that these two unique phenomena contribute to mass production of valuable hydrophobic metabolites by using the Ext-LSI system.

Furthermore, the authors have found that the profile of secondary metabolites in the organic phase in the Ext-LSI system differs considerably from that observed in the aqueous phase in SmC. This finding may provide a route for the enormous number of fungal strains that have so far proved unproductive in traditional screening systems based on SmC to be re-examined for the potential to produce new antibiotics. In this paper, examples of the difference in metabolite profiles found for the SmC and Ext-LSI systems will be presented and a novel interfacial screening system for antibiotic-producing fungi by using the Ext-LSI system introduced.

\section{MATERIALS AND METHODS}

\section{Comparison of secondary metabolites between SmC and Ext-LSI} systems

Microorganisms and media. Two different fungal strains, Penicillium multicolor IAM 7153 and T. atroviride AG2755-5NM398 were used for the comparison of secondary metabolite profile between the SmC and the Ext-LSI systems. The latter is a 6PP-producing mutant prepared with $\mathrm{N}$-methyl- $\mathrm{N}$-nitso- $\mathrm{N}$ nitrosoguanidine..$^{26,29,30}$ Both strains were inoculated in F-1 medium consisted of $20.0 \mathrm{~g}$ of potato starch, $10.0 \mathrm{~g}$ of glucose, $20.0 \mathrm{~g}$ of soy protein (Soypro; Inui Co., Ltd, Osaka, Japan), $1.0 \mathrm{~g}$ of $\mathrm{KH}_{2} \mathrm{PO}_{4}, 0.5 \mathrm{~g}$ of $\mathrm{MgSO}_{4} \cdot 7 \mathrm{H}_{2} \mathrm{O}$ and 1.0 liter of reverse osmosis water, $\mathrm{pH} 6.0$ for the preparation of seed broth. 


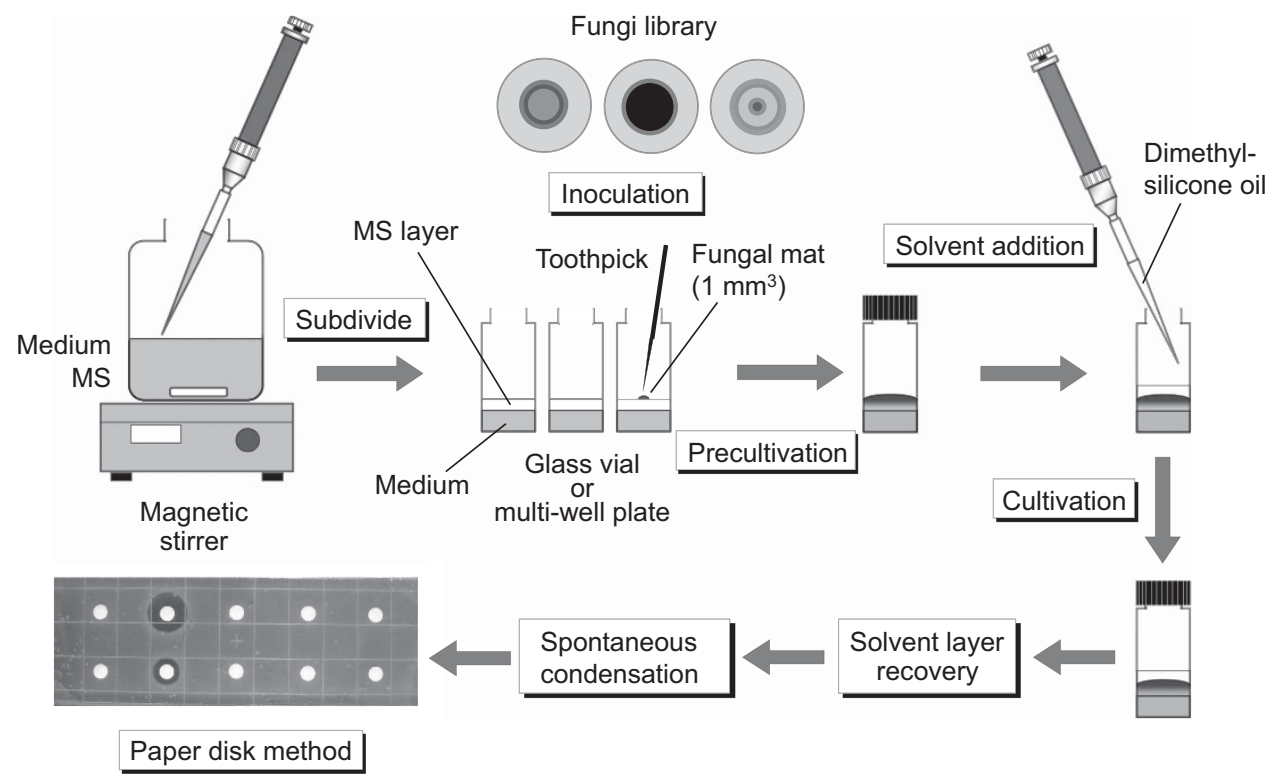

Figure 2 Experimental procedure of a novel screening system of antibiotic-producing fungi by using the Ext-LSI system. A full color version of this figure is available at The Journal of Antibiotics journal online.

Construction, operation and product recovery for SmC. Three-day seed broth ( $375 \mu \mathrm{l}$ ) of $P$. multicolor IAM 7158 was inoculated into $25 \mathrm{ml}$ of potato-dextrose broth prepared in a polypropylene bottle $(100 \mathrm{ml})$. Concerning $T$. atroviride AG2755-5NM398, modified Sabouraud medium, consisted of $40.0 \mathrm{~g}$ of glucose, $10.0 \mathrm{~g}$ of Bacto peptone, $5 \mathrm{mg}$ of $\mathrm{FeSO}_{4} \cdot 7 \mathrm{H}_{2} \mathrm{O}, 20 \mathrm{mg}$ of $\mathrm{MnSO}_{4} \cdot 5 \mathrm{H}_{2} \mathrm{O}, 10 \mathrm{mg}$ of $\mathrm{CaCl}_{2}$ in 1.0 liter of reverse osmosis water, $\mathrm{pH} 6.0$, was used as a liquid medium. Cultivation of both strains was performed at $25^{\circ} \mathrm{C}$ with agitation (200 rpm) for 10 days. After the cultivation, $20 \mathrm{ml}$ of broth was treated by excess $\mathrm{NaCl}$ and extracted with ethyl acetate $(3 \times 20 \mathrm{ml})$. The combined ethyl acetate layer was dehydrated with anhydrous $\mathrm{Na}_{2} \mathrm{SO}_{4}$ and evaporated under reduced pressure, giving a crude mixture of secondary metabolites, which was dissolved in $300 \mu \mathrm{l}$ of 2-propanol. The sample was named SmC-Aq.

Construction, operation and product recovery for Ext-LSI system. Three-day seed broth $(375 \mu \mathrm{l})$, potato-dextrose broth $(25 \mathrm{ml})$ for P. multicolor IAM 7158 or modified Sabouraud medium $(25 \mathrm{ml})$ for T. atroviride AG2755-5NM398 and ballooned microspheres ( $100 \mathrm{mg}$; MMS-DE- 1 ; non-coated type; mean diameter, $30 \mu \mathrm{m}$; density, 0.06; Matsumoto Yushi-Seiyaku, Co., Ltd, Osaka, Japan) were vigorously mixed and poured into a polypropylene beaker $(55 \mathrm{~mm}$ i.d. $\times 70 \mathrm{~mm}$ ). After precultivation at $25^{\circ} \mathrm{C}$ without shaking for 3 days, lowviscosity dimethylsilicone oil ( $25 \mathrm{ml}$; KF-96L-1CS; viscosity, $1 \mathrm{cP}$; b.p., $153^{\circ} \mathrm{C}$; Shin-Etsu Chemical, Co., Ltd, Tokyo) was added onto a fungal mat formed on the surface of a liquid medium. Incubation was continued at $25^{\circ} \mathrm{C}$ without shaking for 7 days. After the cultivation, $20 \mathrm{ml}$ of the organic phase was collected and dried over anhydrous $\mathrm{Na}_{2} \mathrm{SO}_{4}$. The lowly viscous dimethylsilicone oil, KF-96L-1CS, was removed by means of silica gel (60 N; Kanto Chemical, Co., Ltd., Tokyo) column chromatography. KF-96L-1CS was removed by washing with $n$-hexane and the secondary metabolites were eluted with ethyl acetate. The ethyl acetate layer was concentrated under reduced pressure and was dissolved in $300 \mu \mathrm{l}$ of 2-propanol. The sample was named Ext-LSI-Org. The secondary metabolites in the aqueous phase in the Ext-LSI system were recovered by the same manner as SmC-Aq above-mentioned and named Ext-LSI-Aq.

Analysis of secondary metabolites. The profiles of secondary metabolites in three kinds of samples, SmC-Aq, Ext-LSI-Aq and Ext-LSI-Org, were analyzed with high-performance liquid chromatography-photo-diode array detection, LC-2010AHT-SPD-M20A, Shimadzu, Co., Ltd., Kyoto, Japan. The column was Poroshell 120 (4.6 mm i.d. $\times 150 \mathrm{~mm}$; Agilent Technologies, Santa Clara, CA, USA), the column temperature was $40{ }^{\circ} \mathrm{C}$. Each sample $(10 \mu \mathrm{l})$ was analyzed using a 70:30 to $10: 90$ of $0.1 \%$ phosphoric acid-acetonitrile linear gradient for
$40 \mathrm{~min}$ and held to the final solvent mixture for $30 \mathrm{~min}$. The flow rate of the eluent was $0.4 \mathrm{ml} \mathrm{min}^{-1}$ and spectra were monitored at 210 and $254 \mathrm{~nm}$. Spectrophotometric data were collected at $200-500 \mathrm{~nm}$.

\section{Construction of a novel interfacial screening system}

Microorganisms and media. The isolated and stocked filamentous fungi (572 strains), which were restored on potato-dextrose agar plates, were screened for the antibiotic-producing ability with the Ext-LSI system as a cultivation system. Modified Sabouraud medium was used as a liquid medium in the ExtLSI system and Pichia anomala NBRC 10213 was used for antifungal testing. The yeast strain was cultivated on yeast extract peptone dextrose agar plates consisting of $10.0 \mathrm{~g}$ of tryptone, $5.0 \mathrm{~g}$ of yeast extract, $10.0 \mathrm{~g}$ of glucose, $15.0 \mathrm{~g}$ of agar and 1.0 liter of reverse osmosis water, $\mathrm{pH} 6.0$

Procedure of a novel interfacial screening system based on Ext-LSI system. The schematic diagram of a novel interfacial screening system based on the Ext-LSI system is shown in Figure 2. One liter of modified Sabouraud medium and $2.5 \mathrm{~g}$ of MS (MMF-DE-1) were aseptically and vigorously mixed with a magnetic stirrer. Five microliter of the mixture was poured into each glass vessel (volume, $20 \mathrm{ml}$; inside diameter, $24 \mathrm{~mm}$ ) while stirring the mixture. After the formation of MS layer on the surface of a liquid medium, a piece $(\sim 1 \times 1 \mathrm{~mm})$ of each fungal mat was inoculated onto the center of the surface of an MS layer with a toothpick. Precultivation for the formation of the fungusMS mat was done at $25^{\circ} \mathrm{C}$ without shaking for 3 days. After the precultivation, $1 \mathrm{ml}$ of volatile dimethylsilicone oil (KF-96L-0.65CS; viscosity, $0.65 \mathrm{cP}$; Shin-Etsu Chemical, Co., Ltd.) was added onto the fungal mat, and incubation was continued for 7 days. A lid of each vessel was tightly closed for avoidance of volatility of KF-96L-0.65CS. A gas phase of each vessel was aseptically exchanged every other day by introducing $20 \mathrm{ml}$ of air with an auto-pipette. After the incubation for 7 days, the organic phase in the Ext-LSI system was collected and could be spontaneously removed in a fume hood exploiting the volatility of KF-96L-0.65CS (b.p., $100^{\circ} \mathrm{C}$ ). Each condensed sample was dissolved by adding $300 \mu \mathrm{l}$ of ethyl acetate. Thirty microliter of each condensed sample was charged to a paper disk (diameter, $8 \mathrm{~mm}$; thickness, $1.5 \mathrm{~mm}$ ). The paper disk was stored at room temperature overnight to remove ethyl acetate. The disk was put on the surface of a yeast extract peptone dextrose agar plate inoculated with P. anomala NBRC 10213. After preservation at $4{ }^{\circ} \mathrm{C}$ for $4 \mathrm{~h}$ for the diffusion of metabolites into the agar plate, the agar plate was incubated at $30^{\circ} \mathrm{C}$ for $24 \mathrm{~h}$. After the incubation, the diameter of a halo formed was measured with a digital caliper (Figure 2). 

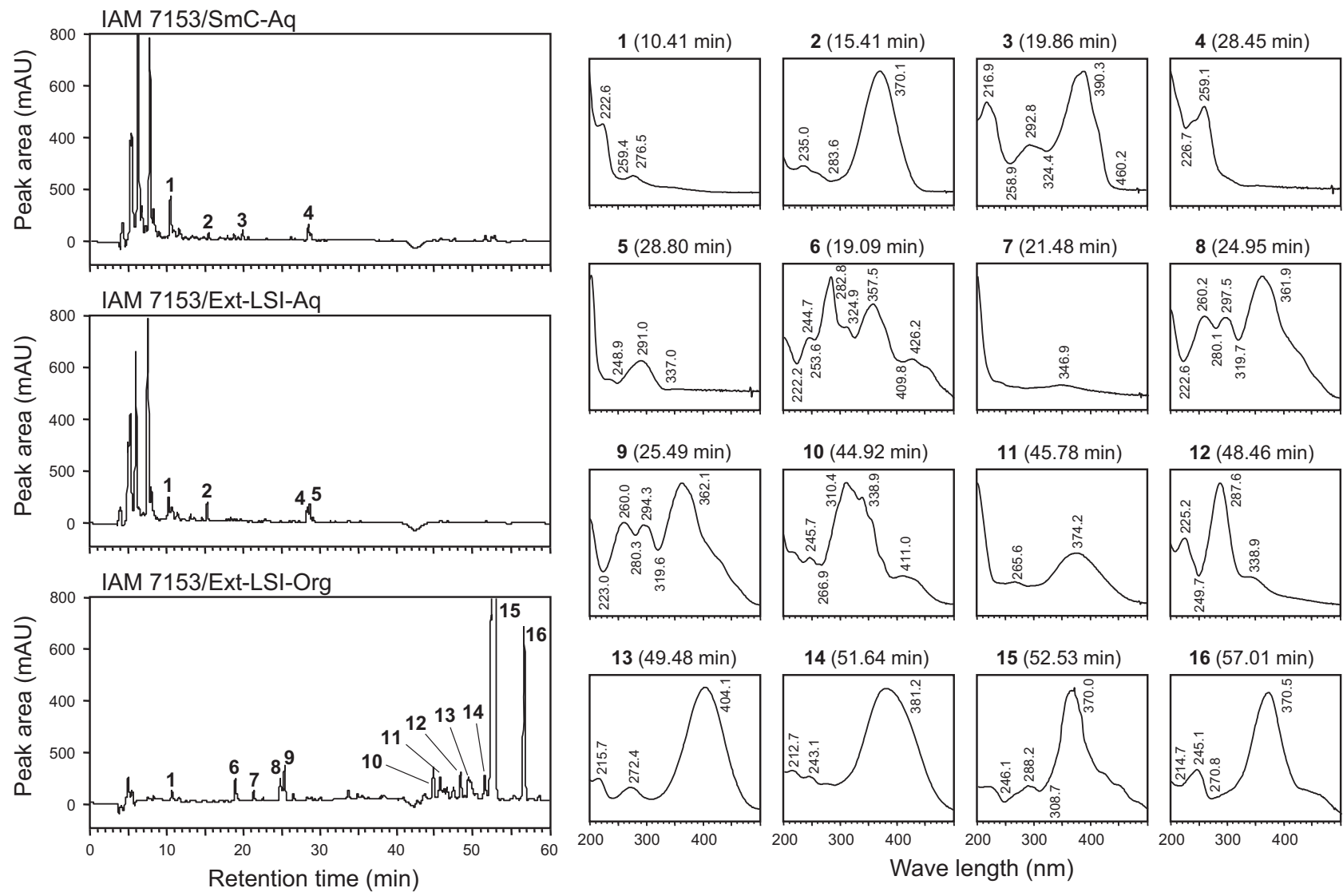

$12(48.46 \mathrm{~min})$
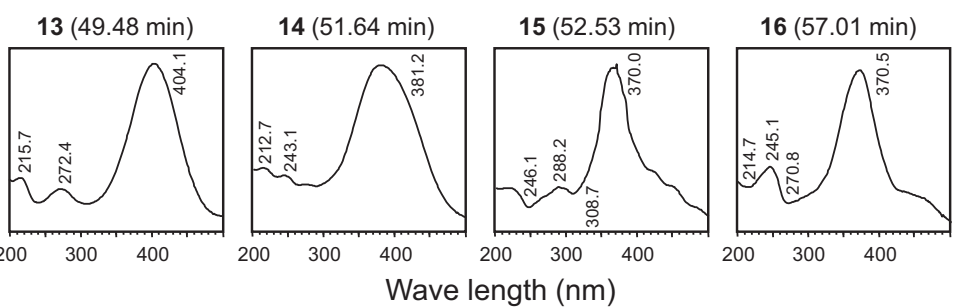

Figure 3 Comparison of profiles of secondary metabolites produced by Penicillium multicolor IAM 7153 between submerged cultivation (SmC) and extractive liquid-surface immobilization (Ext-LSI) system. Aq, metabolites in an aqueous phase; Org, metabolites in an organic phase.

\section{RESULTS AND DISCUSSION}

Comparison of secondary metabolite profiles between $\mathrm{SmC}$ and Ext-LSI systems

First, the metabolite profiles were compared among three kinds of samples, SmC-Aq recovered from an aqueous phase in the SmC, Ext-LSI-Aq from an aqueous phase in the Ext-LSI and Ext-LSI-Org from an organic phase in the Ext-LSI system with P. multicolor IAM 7153. The chromatogram of each sample was monitored at 210 and $254 \mathrm{~nm}$, and the spectrophotometric data were collected at $200-500 \mathrm{~nm}$ by means of a reversed-phase high-performance liquid chromatography-photo-diode array detection. The results are shown in Figure 3. Both samples of SmC-Aq and Ext-LSI-Aq contained relatively hydrophilic metabolites, and the profiles of both samples were similar to each other in spite of very different cultivation systems. On the other hand, remarkable differences were observed in the secondary metabolite profiles between the SmC-Aq and Ext-LSI-Org samples (Figure 3). In the organic phase in the Ext-LSI system (Ext-LSI-Org), many hydrophobic metabolites, such as peaks 10-16, were detected. The maximal absorption wavelength $\left(\lambda_{\max }\right)$ of the metabolites except peak 12 was over $300 \mathrm{~nm}$. Especially, peaks 15 and 16 were predominant and exhibited unique UV-Vis patterns. The UV-Vis patterns are similar to those of azaphilones such as sclerotiorin $^{31}$ and isochromophilones. ${ }^{32}$

Next, the metabolite profiles were compared among SmC-Aq, Ext-LSI-Aq and Ext-LSI-Org from T. atroviride AG2755-5NM398. As shown in Figure 4, the profiles of hydrophilic metabolites in SmC-Aq and Ext-LSI-Aq were different to each other. Four peaks 3-6 were detected in only Ext-LSI-Aq. Especially, peak 3 was predominant compared with other peaks in Ext-LSI-Aq. A fungicidal secondary metabolite 6PP (peak 2) was detected in both SmC-Aq and Ext-LSI-Aq samples. On the other hand, Ext-LSI-Org contained unique hydrophobic metabolites (peaks 7-9). The UV-Vis pattern of peak 7 was similar to that of 6PP (peak 2). Both of peaks 8 and 9 did not exhibit unusual UV-Vis spectra. Thus, as for T. atroviride AG2755-5NM398, peculiar hydrophilic and hydrophobic metabolites were gained in the Ext-LSI system (Figure 4).

It is well known that the differentiation of fungi is closely linked to the production of secondary metabolites. ${ }^{33}$ For example, while some secondary metabolites such as linoleic acid derivatives (psi factors) activate sporogenesis, ${ }^{34,35}$ the sporogenesis often triggers the production of secondary metabolites such as pigments. ${ }^{36,37}$ It was also reported that Penicillium brevicompactum could produce mycophenolic acid only after formation of aerial hyphae. ${ }^{38}$

Although the filamentous form was predominant in SmC of P. multicolor IAM 7153 and T. atroviride AG2755-5NM398, both strains formed a fungal mat developing much aerial hyphae and spores on an interface between an organic phase and a liquid medium in the Ext-LSI system. Thus, it is possible that the morphological difference between the SmC and the Ext-LSI systems brings about the difference of the profile of secondary metabolite between the SmC and the Ext-LSI systems.

Moreover, an organic phase in the Ext-LSI system has two significant roles in the spontaneous extraction of hydrophobic secondary metabolites from fungal cells and the supply of oxygen from atmosphere. The former role maybe contribute to the alleviation of product and/or feed-back inhibitions by secondary metabolites. 

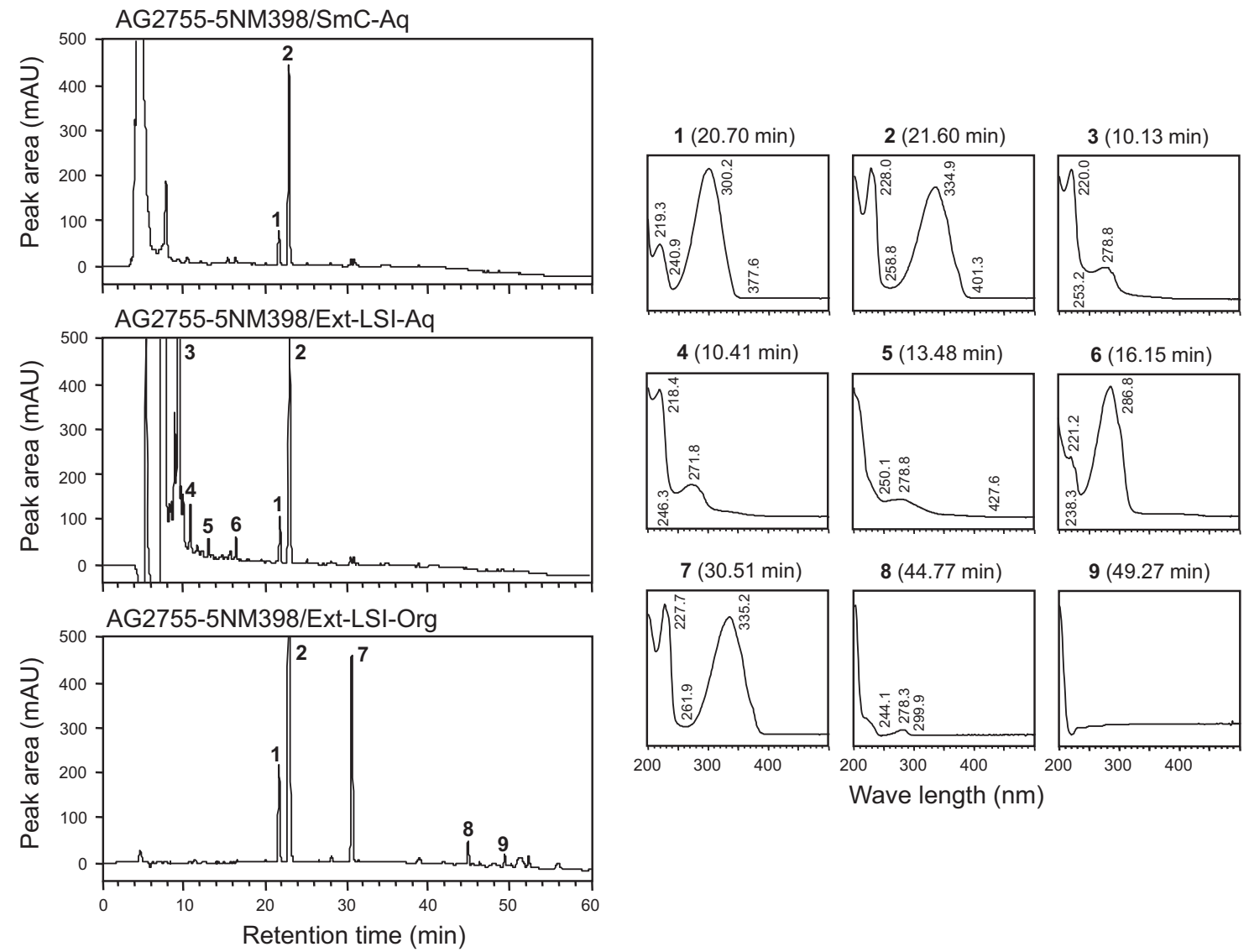

Figure 4 Comparison of profiles of secondary metabolites produced by Trichoderma atroviride AG2755-5NM398 between submerged cultivation (SmC) and extractive liquid-surface immobilization (Ext-LSI) system. Aq, metabolites in an aqueous phase; Org, metabolites in an organic phase.

Indeed, it has been observed that the accumulation of a fungicidal secondary metabolite (6PP) of which MICs against some fungi are $0.1-0.5 \mathrm{gl}^{-1}$ reaches $7.1 \mathrm{gl}^{-1}$ in the organic phase in the Ext-LSI system. ${ }^{26}$ The excellent productivity of $6 \mathrm{PP}$ was also observed in this study as shown in Figure 3. The organic phase in the liquid-liquid interface bioreactor also has an extractant and a reservoir of toxic substrate and/or product to hold very high level of various hydrophobic conversion products. ${ }^{17,20-25}$ Thus, the spontaneous extraction of hydrophobic secondary metabolites maybe contribute to peculiarity of secondary metabolite profile of the Ext-LSI-Org.

Next, it is also assumed that high solubility of oxygen in the organic phase affects the peculiar metabolite profile in the Ext-LSI-Org. It is well known that dissolved oxygen affects fungal growth, morphology and metabolite production. ${ }^{39,40}$ It was also reported that the enhancement of dissolved oxygen tension in a broth led to the increase of mycelia aggregate diameter and the accumulation of ganoderic acid, ${ }^{39}$ whereas the decrease of dissolved oxygen tension led the formation of weak and fluffy pellets. ${ }^{40}$

Generally, organic solvents dissolve a lot of oxygen compared with water. Indeed, $n$-alkanes such as $n$-hexadecane, ${ }^{41}$ perfluorocarbon ${ }^{42}$ and dimethylsiloxane $\mathrm{e}^{43,44}$ have been used as an oxygen vector for the elevation of dissolved oxygen in fermentation, bioconversion and enzyme production. The low-density dimethylsilicone oil (KF-96L-1CS) used by us could also act as an oxygen vector to enhance the production of metabolite (technical information by Shin-Etsu Chemical, Co., Ltd; http://dcproducts.com.au/documents/ SiliconeFluids.pdf). It should be noted that the mass transfer in the dimethylsilicone oil is higher than for the previously mentioned organic solvents because of the lower viscosity (1 cP) of KF-96L-1CS.

In addition to above-mentioned characteristics of the Ext-LSI system, it should be noted that CCR is effectively alleviated in the system $^{30}$ like solid-state cultivation. ${ }^{45,46}$ The CCR is an unfavorable phenomenon disturbing the production of metabolites, enzymes and biomass, ${ }^{47,48}$ and appears by adding only $1 \%$ easily metabolizable carbon source such as glucose, sucrose and glycerol. ${ }^{49}$ Although the production of 6PP by T. atroviride AG2755-5NM398 was completely inhibited by adding over $10 \%(\mathrm{w} / \mathrm{v})$ of glucose and fructose in the SmC, the CCR was effectively derepressed even in the presence of $25 \%(\mathrm{w} / \mathrm{v})$ glucose and fructose in the Ext-LSI system. ${ }^{30}$ Thus, the derepression of CCR in the Ext-LSI system may be favorable to the production of hydrophobic secondary metabolites.

In conclusion, Ext-LSI enables the production of many different hydrophobic and hydrophilic metabolites compared with those found by SmC. Especially, the authors view as the important diversity of hydrophobic metabolites rather than that of hydrophilic ones because of the difficulty of efficient production of the hydrophobic metabolites in SmC and easy elevation of hydrophilicity of the hydrophobic metabolites via microbial transformation such as hydroxylation and glycosylation using the liquid-liquid interface bioreactor system. The SmC extracts analyzed in this study were whole-culture ethyl acetate extracts. Further insights into the differences between the SmC and the Ext-LSI systems may be gained by separating cells and a supernatant in the SmC, and extracting their associated secondary metabolite content separately. This will be the subject of a future study. 


\section{Construction of a novel interfacial screening system}

As stated above, the Ext-LSI system has some unique and valuable characteristics for the production of secondary metabolites as follows: (i) unique secondary metabolite profiles different from those in SmC, (ii) straightforward morphological control of fungi to a mat form, (iii) alleviation of product and/or feed-back inhibitions by spontaneous extraction of hydrophobic metabolites, (iv) efficient supply of oxygen from the organic phase to fungal cells and (v) derepression of the CCR. Indeed, some unique hydrophobic secondary metabolites are much accumulated in an organic phase in the Ext-LSI system (Figures 3 and 4).

Next, the authors tried to apply the Ext-LSI system to a novel highthroughput screening system for the selection of antibiotic-producing fungi. The procedure is summarized in Figure 2. A piece of each restored fungal mat (572 strains) was inoculated on the surface of an MS layer formed on a liquid medium $(5 \mathrm{ml})$ with a toothpick. After 3 -day precultivation at $25^{\circ} \mathrm{C}$ without shaking, $1 \mathrm{ml}$ of volatile dimethylsilicone oil, KF-96L-0.65CS, was added onto a fungal mat containing MS formed on a liquid medium. The dimethylsilicone oil is highly volatile (b.p., $100^{\circ} \mathrm{C}$ ) and is entirely harmless against almost all fungi. After 7-day cultivation, the organic phase was collected and spontaneously condensed in a draft. Thus, troublesome solvent extraction and removal of solvent were eliminated in the screening system. Each condensed sample was dissolved by adding $300 \mu \mathrm{l}$ of ethyl acetate and applied to paper disk method for the evaluation of antifungal activity against Pichia anomala NBRC 10,213. As a result, 125 samples exhibited significant antifungal activity (21.9\%).

In conclusion, the Ext-LSI system can afford many unique secondary metabolite profiles different from those in SmC. Especially, hydrophobic metabolites are efficiently accumulated in the organic phase in the system. The alleviation of product and/or feed-back inhibitions, efficient supply of oxygen from the organic phase to fungal cells and the derepression of CCR are also valuable characteristics of the Ext-LSI system.

Moreover, it was verified that the Ext-LSI system is useful for the high-throughput screening of antibiotic-producing fungi. The antifungal hit rate of the interfacial screening system reached $21.9 \%$ against $P$. anomala NBRC 10,213. Nowadays, hazardous pathogens, such as multidrug-resistant pathogens and harmful viruses, are gradually expanding worldwide. However, research and development of new antibiotic by pharmaceutical companies markedly reduced. The authors expect that the novel interfacial screening system above-mentioned is widely applied, many new antimicrobial metabolites are discovered, and many antibiotics effective in multidrugresistant pathogens and harmful viruses are developed.

\section{ACKNOWLEDGEMENTS}

The authors wish to thank Matsumoto Yushi-Seiyaku, Co., Ltd, for the kind gift of microspheres. This work was supported by a Grant-in-Aid for Science Research (C) (No. 25450115) from the Japan Society for the Promotion of Science.

1 Taiwo, S. S. Antibiotic-resistant bugs in the 21th century: a public health challenge. World J. Clin. Infect. Dis. 1, 11-16 (2011).

2 Bereket, W. et al. Update on bacterial nosocomial infections. Eur. Rev. Med. Pharmacol. Sci. 16, 1039-1044 (2012).

3 Bérdy, J. Bioactive microbial metabolites. J. Antibiot. 58, 1-26 (2005).

4 Keller, N. P., Turner, G. \& Bennet, J. W. Fungal secondary metabolism-from biochemistry to genomics. Nat. Rev. Microbiol. 3, 937-947 (2005).

5 Kumar, S. \& Kaushik, N. Metabolites of endophytic fungi as novel source of biofungicide: a review. Phytochem. Rev. 11, 507-522 (2012).
6 Aly, A. H., Debbab, A., Kjer, J. \& Proksch, P. Fungal endophytes from higher plants: a prolific source of phytochemicals and other bioactive natural products. Fungal Diversity 41, 1-16 (2010)

7 Isaka, M., Palasarn, S., Kocharin, K. \& Saenboonryeng, J. A cytotoxic xanthone dimer from the entomopathogenic fungus Aschersonia sp. BCC 8401. J. Nat. Prod. 68 945-946 (2005).

8 Kagamizono, T. et al. Bassiatin, a new platelet aggregation inhibitor produced by Beauveria bassiana K-717. J. Antibiot. 48, 1407-1412 (1995).

9 Lee, Y. M. et al. Marine-derived Aspergillus species as a source of bioactive secondary metabolites. Mar. Biotechnol. 15, 499-519 (2013).

10 Yu, Z., Lang, G., Kajahn, I., Schmaljohann, R. \& Imhoff, J. F. Scopularides A and B cyclodepsipeptides from a marine sponge-derived fungus, Scopulariopsis brevicaulis. J. Nat. Prod. 71, 1052-1054 (2008)

11 Du, L.-X., Jia, S.-J. \& Lu, F.-P. Morphological changes of Rhizopus chinensis 12 in submerged culture and its relationship with antibiotic production. Process Biochem. 38, 1643-1646 (2003).

12 Higashiyama, K., Murakami, K., Tsujimura, H., Matsumoto, N. \& Fujikawa, S. Effects of dissolved oxygen on the morphology of an arachidonic acid production by Mortierella alpina 1S-4. Biotechnol. Bioeng. 63, 442-448 (1999).

13 Bacon, C. W., Sweeney, J. G., Robbins, J. D. \& Burdick, D. Production of penicillic acid and ochratoxin A on poultry feed by Aspergillus ochraceus: temperature and moisture requirements. Appl. Microbiol. 26, 155-160 (1973).

14 Zhang, Z. Y., Jin, B. \& Kelly, J. M. Effects of cultivation parameters on morphology of Rhizopus arrhizus and the lactic acid production in a bubble column reactor. Eng. Life Sci. 7, 490-496 (2007).

15 Morrin, M. \& Ward, O. P. Relationships between fungal growth, morphology and fumaric acid production by Rhizopus arrhizus. Mycol. Res. 94, 505-510 (1990).

16 Liao, W., Liu, Y. \& Chen, S. Studying pellet formation of a filamentous fungus Rhizopus oryzae to enhance organic acid production. Appl. Biochem. Biotechnol. 137-140, 689-701 (2007).

17 Oda, S. \& Isshiki, K. Liquid-surface immobilization system and liquid-liquid interface bioreactor: application to fungal hydrolysis. Process Biochem. 42, 1553-1560 (2007).

18 Ozeki, K., Takagi, Y., Oda, S. \& Ohashi, S. Production of xylanase with a transformant of Aspergillus oryzae RIB40 in a liquid-surface immobilization (LSI) system. J. Biosci. Bioeng. 109, 224-226 (2010).

19 Ozeki, K. et al. Production of $\beta$-glucosidase by a transformant of Aspergillus oryzae RIB40 in a liquid-surface immobilization (LSI) system. J. Biol. Macromol. 11, 23-30 (2011)

20 Oda, S., Wakui, H. \& Ohashi, S. Efficient hydrolytic reaction of an acetate ester with fungal lipase in a liquid-liquid interface bioreactor ( $L-L$ IBR) using $\mathrm{CaCO}_{3}$-coated ballooned microsphere. J. Biosci. Bioeng. 112, 151-153 (2011).

21 Oda, S. \& Isshiki, K. Asymmetric reduction of benzil to (S)-benzoin with Penicillium claviforme IAM 7294 in a liquid-liquid interface bioreactor (L-L IBR). Biosci. Biotechnol. Biochem. 72, 1364-1367 (2008).

22 Oda, S., Isshi, K. \& Ohashi, S. Regio- and stereoselective subterminal hydroxylation of $n$-decane by fungi in a liquid-liquid interface bioreactor (L-L IBR). Bull. Chem. Soc. Jpn. 82, 105-109 (2009).

23 Oda, S., Sakamoto, N., Horibe, H., Kono, A \& Ohashi, S. Enhancement of $n$-decane hydroxylation activity of Monilliera sp. NAP 00702 in a liquid-liquid interface bioreactor by mixing of anion-exchange resin microparticles. Process Biochem. 47, 2494-2499 (2012).

24 Oda, S., Sakamoto, N., Horibe, H., Kono, A. \& Ohashi, S. Relationship between interfacial hydrophobicity and hydroxylation activity of fungal cells located on an organic-aqueous interface. J. Biosci. Bioeng. 115, 544-546 (2013).

25 Oda, S., Fujinuma, K., Inoue, A. \& Ohashi, S. Synthesis of (-)- $\beta$-caryophyllene oxide via regio- and stereoselective endocyclic epoxidation of $\beta$-caryophyllene with Nemania aenea SF 10099-1 in a liquid-liquid interface bioreactor (L-L IBR). J. Biosci. Bioeng. 112, 561-565 (2011).

26 Oda, S., Isshiki, K. \& Ohashi, S. Production of 6-pentyl- $\alpha$-pyrone with Trichoderma atroviride and its mutant in a novel extractive liquid-surface immobilization (Ext-LSI) system. Process Biochem. 44, 625-630 (2009).

27 Cooney, J. M., Lauren, D. R., Poole, P. R. \& Whitaker, G. Microbial transformation of the Trichoderma metabolite 6-n-pentyl-2H-pyran-2-one. J. Nat. Prod. 60, 1242-1244 (1997).

28 Parker, S. R., Cutler, H. G., Jacyno, J. M. \& Hill, R. A. Biological activity of 6-pentyl-2Hpyran-2-one and its analogs. J. Agric. Food Chem. 35, 2774-2776 (1997).

29 Oda, S. et al. Enhancemnet of 6-pentyl- $\alpha$-pyrone fermentation activity in an extractive liquid-surface immobilization (Ext-LSI) system by mixing anion-exchange resin microparticles. J. Biosci. Bioeng. 114, 596-599 (2012).

$30 \mathrm{Oda}, \mathrm{S}$., Araki, H. \& Ohashi, S. Derepression of carbon catabolite repression in an extractive liquid-surface immobilization (Ext-LSI) system. J. Biosci. Bioeng. 113, 742-745 (2012).

31 Natsume, M., Takahashi, Y. \& Marumo, S. Clamydospore-like cell-inducing substances of fungi: close correlation between chemical reactivity with methylamine and biological activity. Agric. Biol Chem. 52, 307-312 (1988).

32 Arai, N. et al. Isochromophilones IIIVI, inhibitors of acyl-CoA:cholesterol acyltransferase produced by Penicillium multicolor FO-3216. J. Antibiot. 48, 696-702 (1995).

33 Calvo, A. M., Wilson, R. A., Bok, J. W. \& Keller, N. P. Relationship between secondary metabolism and fungal development. Microbiol. Mol. Biol. Rev. 66, 447-459 (2002).

34 Champe, S. P., Rao, P. \& Chang, A. An endogenous inducer of sexual development in Aspergillus nidulans. J. Gen. Microbiol. 133, 1383-1387 (1987).

35 Champe, S. P. \& El-Zayat, A. A. E. Isolation of a sexual sporulation hormone from Aspergillus nidulans. J. Bacteriol. 171, 3982-3988 (1989). 
36 Alspaugh, J. A., Perfect, J. R. \& Heitman, J. Cryptococcus neoformans mating and virulence are regulated by the G-protein $\alpha$ subunit GPA1 and cAMP. Genes Dev. 11 3206-3217 (1997).

37 Kawamura, C., Tsujimoto, T. \& Tsuge, T. Targeted disruption of a melanin biosynthesis gene affects conidial development and UV tolerance in the Japanese pear pathotype of Alternaria alternata. Mol. Plant Microbe Interact. 12, 59-63 (1999).

38 Bartman, C. D. et al. Mycophenolic acid production by Penicillium brevicompactum on solid media. Appl. Environ. Microbiol. 41, 729-736 (1981).

39 Tang, Y.-J. \& Zhong, J.-J. Role of oxygen supply in submerged fermentation of Ganoderma lucidum for production of Ganoderma polysaccharide and ganoderic acid. Enzyme Microb. Technol. 32, 478-484 (2003).

40 Cui, Y. Q., van der Lans, R. G. J. M. \& Luyben, K. C. A. M. Effects of dissolved oxygen tension and mechanical forces on fungal morphology in submerged fermentation. Biotechnol. Bioeng. 57, 409-419 (1998).

$41 \mathrm{Li}$, M., Meng, X., Diao, E., Du, F. \& Zhao, X. Productivity enhancement of $S$-adenosylmethionine in Saccharomyces cerevisiae using $n$-hexadecane as oxygen vector. J. Chem. Technol. Biotechnol. 87, 1379-1384 (2012).

42 Amaral, P. F. F., Rocha-Leão, M. H. M., Marrucho, I. M., Coutinho, J. A. P. \& Coelho, M. A. Z. Improving lipase production using a perfluorocarbon as oxygen carrier. J. Chem. Technol. Biotechnol. 81, 1368-1374 (2006).
43 Quijano, G., Revah, S., Gutiérrez-Rojas, M., Flores-Cotera, L. B. \& Thalasso, F. Oxygen transfer in three-phase airlift and stirred tank reactors using silicone oil as transfer vector. Process Biochem. 44, 619-624 (2009).

44 Ates, S., Dingil, N., Bayraktar, E. \& Mehmetoglu, U. Enhancement of citric acid production by immobilized and freely suspended Aspergillus niger using silicone oil. Process Biochem. 38, 433-436 (2002).

45 Viniegra-González, G. \& Favela-Torres, E. Why solid-state fermentation seems to be resistant to catabolite repression? Food Technol. Biotechnology 44, 397-406 (2006).

46 Lu, L.-P., Zhang, B.-B. \& Xu, G.-R. Efficient conversion of high concentration of glycerol to Monacolin K by solid-state fermentation of Monascus purpureus using bagasse as carrier. Bioprocess Biosyst. Eng. 36, 293-299 (2013).

47 Hölker, U., Höfer, M. \& Lenz, J. Biotechnological advantages of laboratory-scale solid-state fermentation with fungi. Appl. Microbiol. Biotechnol. 64, 175-186 (2004).

48 Aguilar, C. N., Augur, C., Favela-Torres, E. \& Viniegra-González, G. Induction and repression patterns of fungal tannase in solid-state and submerged cultures. Process Biochem. 36, 565-570 (2001).

49 de Souza, D. F., de Souza, C. G. M. \& Peralta, R. M. Effect of easily metabolizable sugars in the production of xylanase by Aspergillus tamarii in solid-state fermentation. Process Biochem. 36, 835-838 (2001). 